\title{
Emerging Trends in Virtual Reality for Gaming: an assessment of best practices from research and development in the gaming industry
}

\author{
Dr. Jason Nolan, Daniel Harley (RE/Lab, Ryerson University)
}

Anthony Walsh, Eric McQuiggan (Phantom Compass Inc.)

Completed with funding support from the Ontario Centres of Excellence TalentEdge program (OCE) and the Ontario Media Development Corporation (OMDC). Infrastructure funding supporting this work has been provided by Canadian Foundation for Innovation, Ontario Ministry of Research and Innovation and Ryerson University.

\begin{abstract}
Consumer virtual reality is a new and rapidly changing medium, with best practices still emerging across the industry. As part of an industry-academic collaboration, Phantom Compass partnered with Ryerson's Responsive Ecologies Lab to design and playtest three virtual reality prototypes that employ and expand on the current best practices. This white paper collates industry research and development focusing on player experience and comfort, and summarizes the results of our design process.
\end{abstract}

Keywords: Virtual Reality, Simulator Sickness, Player Experience Design, Game Design. 


\section{Introduction}

The first wave of consumer virtual reality (VR) hardware and software has resulted in a new market expected to be valued in the billions of dollars within the next five to ten years (Riccitiello, 2016). While gaming is the first market for consumer VR, several other VR markets are predicted to grow in the coming years, including those related to social media, sports, medicine, and education (Heller, 2015). For each of these markets to become successful, they must address the potential side effects of VR, including the many factors that contribute to simulation sickness.

As part of an industry-academic collaboration, Phantom Compass maintains an ongoing partnership with the Responsive Ecologies Lab (RE/Lab) at Ryerson University, and previously the Experiential Design and Gaming Environments (EDGE) Lab. This present collaboration focused on researching and developing user-tested design strategies for interactive VR. Over the course of a four-month project, we created three VR game-like experiences to test and expand current practices for VR design, refining each prototype based on user feedback and lessons learned throughout our research.

Accordingly, this white paper includes a collection of best practices from a number of industry sources focusing on player experience and comfort, as well as the summarized results of our iterative design process. This paper is not intended as a comprehensive guide to VR design: many of the design recommendations cited throughout this paper are from sources that are not easily verified as their methodology is either unknown or unclear. Furthermore, many of the sources should be understood as individual cases, with the potential for different results under different conditions (i.e., depending on the particular requirements of the game and the headset). 
We view this work as a documentation of our process and our lessons learned as we strive to improve player comfort within a rapidly changing medium.

\section{Simulator Sickness}

As we interact with the world around us, our visual, vestibular, and proprioceptive systems send sensory information to our brain to help maintain our balance and make sense of the world. VR can create a conflict between these systems, providing visual information that does not accurately match the information from our vestibular and proprioceptive systems. This mismatch can lead to simulator sickness, which refers to any combination of symptoms related to nausea, fatigue, and disorientation (Hamm \& Padget, 2016; Malaika, 2015; Oculus, 2016a). Depending on the player and the design, these symptoms can be exacerbated by a number of factors, including visual discrepancies, perceived motion, flickering, brightness, high latency, as well as the weight and heat of the headset (Lewis-Evans, 2015).

While headsets and hardware can contribute to simulator sickness with their limited field of view, limited depth of focus, and limited image quality (Abrash, 2015), recent rhetoric from Oculus and Valve suggests that it is "no longer the hardware's fault" (Faliszek, 2015), and "Developers are responsible for ensuring their content conforms to all standards and industry best practices on safety and comfort, and for keeping abreast of all relevant scientific literature on these topics" (Oculus, 2016a). This responsibility will be an ongoing challenge for VR designers and developers, with hardware development in a competitive growth phase, minimal standardization across platforms, and an evolving set of best practices.

The most common recommendations to mitigate possible discomfort are to avoid ingame movement and maintain visual consistency. Visual consistency applies to such aspects as 
frame rate optimization, maintaining a stable horizon line, and providing static visual reference points (e.g., a cockpit and a static skybox; Oculus, 2016a; Unity, 2016). In-game movement refers to any perceived change in player speed or direction.

Current headsets and controllers track head and hand movement; any other movement that does not match what the player is physically doing can lead to discomfort. Beyond avoiding all non-tracked movement, developers can limit movement to forms that involve linear, constant forward momentum (Carmack, 2015; Oculus, 2016a). Any involuntary sense of motion should be avoided, including the perceived motion caused by, for example, moving textures or peripheral movement (Lewis-Evans, 2015). For more detail, see the section on Player Movement.

There is no one-size-fits-all solution to fully eliminate the side effects of VR, putting limitations on the type and complexity of VR experiences, and muting some of its marketed promise. It is possible that players will develop 'VR legs' over time, but not within the span of a single play session or a single game (Reimer \& Schwartz, 2014). Players will have varying susceptibilities to the causes of simulator sickness, and will not necessarily be able to identify the cause of their discomfort. Design that allows for or encourages frequent breaks could mitigate negative effects (Martz, 2016), and may also help mitigate the possibility of "disembarkation sickness," which refers to the body's readjustment to the conditions of the real world after VR (Oculus, 2016a).

\section{Player Comfort}

Designing for player comfort is essential in VR as new players will not know their tolerance levels and will not know how VR might affect their homeostatic balance (Forsyth, 
2014). VR is real enough to create physical, psychological, and social discomfort (Voll, 2016a, 2016b). Default configurations should strive to apply strategies that mitigate or eliminate these forms of discomfort while also informing players of the potential risks of the experience.

\section{Physical Comfort}

As a baseline for physical comfort, developers must take into account differences in players' bodies, abilities, and environments (Malaika, 2015) to ensure that the individual user is taken into account. When players begin a VR experience, they will need time to orient themselves, adjust, and familiarize themselves with the interactions (West, 2015). Design that respects a neutral head position will be most comfortable, with core gameplay occurring within 144 degrees horizontally, 60 degrees above the player's eye line and 40 degrees below (Ekanayake, 2016). As a general rule, it is best to avoid exhausting head movement or gameplay that forces players to interact and look in 360 degrees (Luckey, 2014). Configurable options for aspects such as movement style or brightness will help to address differences in susceptibilities to simulator sickness — what works for one player may not work for another (Joudrey, 2016).

\section{Psychological comfort}

VR is real enough to create psychological trauma, and the contract between player and developer should involve trust and transparent communication: the game must clearly indicate what type of experience it offers before the player begins (Voll, 2016). The Oculus comfort ratings (comfortable, moderate, and intense) primarily refer to physical comfort, as the clearest difference between comfortable and intense is the amount of motion and camera movement. Developers and designers must also understand (and clarify to players) when the content is likely to be unpleasant, frightening, or containing anything that could trigger a phobia like heights, 
claustrophobic rooms, or underwater environments (Metcalfe, Alge, Faaborg, Dearman \& Pullen, 2016).

\section{Social comfort}

A player's social comfort is closely related to their physical and psychological comfort in VR. Designing safe zones and places for players to stop, rest, and potentially remove their headset will help contribute to physical comfort, while also functioning as a way for players to easily exit a stressful social environment (Voll, 2016a). Players must be able to maintain full control over their avatar, their field of view, and motion (Podwal, Welker, Clement \& Tilton, 2016). In social environments, this can be extended by limiting what players are able to do to each other. Some bullying in VR might be prevented by not allowing players to obstruct another player's view or force movement in any way (Podwal, Welker, Clement \& Tilton, 2016). Players will have a real psychological response to virtual harassment, making it necessary to provide rules or options that encourage safe, comfortable experiences. For example, the social app Altspace VR (2016) offers an invisible 'Space Bubble' that other avatars cannot enter to help ensure that players respect physical boundaries and personal space.

\section{Player Movement}

As one of the main causes of the mismatch between what the player sees and feels, ingame movement is a fundamental design challenge for VR. O'Luanaigh (2016) reports that 40$50 \%$ of players suffer discomfort when walking in VR with traditional controls, which includes many forms of movement that are common in games, e.g., strafing, backing up, or rotating. As mentioned in the previous sections, some visual strategies can help to mitigate discomfort. Static reference points (e.g., a cockpit, a static skybox) can help to hide or reduce the sense of motion 
(Oculus, 2016a). Or, when it is necessary to create a sense of movement, visual cues like particle effects and speed trails can help give an impression of motion without increasing the speed (Unity, 2016). Broader movement strategies fall under two overlapping categories, affecting the design of in-game movement options and affecting the use of the in-game camera.

\section{Camera}

With head-tracking, the camera in VR is controlled by the player's look direction. Any camera movement is equivalent to player movement, implying that any movement that is not controlled by the player can lead to discomfort. If objects in the world are moving, the camera's height and position can create a sense of motion, particularly when the objects are close to the player's field of view or in the player's periphery. When it is necessary to move the camera, consistency and predictability are integral, and can be paired with efforts to limit the amount of movement (Link \& Vasconcellos, 2016). For example, if the player's avatar jumps to a platform, the camera could follow only after the avatar reaches the intended height (Hurd \& Reiland, 2016). A player jumping up and down in third person would not affect the camera's position.

For particularly uncomfortable movement like pitch, yaw or roll, visual cues and anticipatory motion can be applied to in-game objects such as a car or cockpit rather than the camera itself (Meyer, 2016; Oculus, 2016a). Other uncomfortable camera movement, like rotation, can be mitigated by increasing the speed beyond what is immediately perceivable, or by dynamically reducing the field of view to decrease the overall sense of motion (Lloyd \& Thompson). Solutions like these require testing in order to create a comfortable default configuration that can later be tweaked by the player.

\section{Movement options}


Players are relatively comfortable with forward movement at a constant velocity, with real world human speeds being better for longer periods (e.g., a consistent walking or jogging pace; Oculus, 2016a). As with the camera motion, the duration and magnitude of the movement must be limited, with the duration being more significant than the magnitude: motion can be very fast for brief moments (Lloyd \& Thompson, 2016). To reduce or eliminate the sense of motion, several VR movement options have been proposed (see e.g., Colgan, 2015). Each has its limitations; movement may be a problem that will require adjustment or reconsideration for each new game (Foster, 2015).

Teleportation could be the safest compromise, providing the ability to cross distances without a visual sense of locomotion. However, without clear reference points, a stable POV, and visual cues, teleportation can also be disorienting (Malaika, 2015). The visual cues can be in the environment (e.g. a statue in the distance, a teleportation disk that the player tosses or shoots ahead) as well as in the user interface (UI) (e.g. a grid showing what direction the player will end up). In some cases, the teleportation points can be fixed, placing the player in a position in view of a point of interest or in the centre of the action (Whiting \& Donaldson, 2016).

\section{Player Experience Design}

As players often have complete control over the camera and look direction, one of the challenges of VR design is to appropriately direct the player's experience (Harris, 2015). A common solution is to offer one or two points of interest that are clear and easy to follow, allowing the player time to safely explore their surroundings (Brillhart, 2016; Unseld, 2015). For now, short games and demos are the norm, as brief sharable moments can be an opportunity for players to introduce VR to new users (Gaudechon \& Liu, 2015). VR experiences range from 
those in which the player is a passive observer to those in which the player is an active participant who will have an active effect on the game world (Shamlin, 2016). In each of these experiences, player experience design will consist of a balance between what players want to do and what they are able to do. In addition, most current players are novice users who will need help understanding the possibilities and the limitations of VR (Duringer, 2016).

\section{The Possibilities of VR}

Players often expect to be able to do things that they can do in reality or in other games (Whiting \& Donaldson, 2016). With hands in VR, players expect to touch objects, which can lead to an expectation to lift and then throw the object. To help anticipate user actions, 'brownboxing' can be part of the design process, i.e., creating 3D props out of cardboard to observe how players try to solve puzzles with the objects at hand (Schell, 2016).

Within the game, players can be guided implicitly and explicitly. Explicit player guidance can take the form of tutorials and/or helpful Non-Player Characters (NPC) who direct player actions or player gaze (West, 2015). When an NPC looks or points in a direction, players will often follow the indicated direction. Implicit player guidance can take the form of simplifying ingame actions that players might otherwise be required to learn or master. 'Helper systems' can mitigate the possibility of simulator fatigue, replacing a realistic gesture with a simplified gesture (Foster, 2015). The system could maximize the ease of grasping an object while minimizing the number of required learned actions. Any such system will require appropriate visual, aural, and/or haptic feedback to reinforce necessary actions and to satisfy player expectations.

\section{The Limitations of VR}

The increase in expectations can also lead to an increase in frustration when actions in VR are not rewarded. One of the primary hurdles here is tracking. Currently only the headset and 
controllers (not the player's full hands) are tracked, meaning that players must learn what is possible within the constraints of an unfamiliar system. Players will also be limited by their tracking space, which will not match the 'physical' bounds of the virtual environment. Although players might generally avoid putting their hands or body through virtual objects (as reaching through hard virtual surfaces can be uncomfortable for some players; Oculus, 2016a), visual cues and feedback can help to discourage unintended actions (Montoya \& Sproll, 2016). Oculus recommends a gradual fade to black if players leave the tracking zone (2016).

A proper sense of scale can help to situate players within the game world, but designers must ensure that all interactive objects are at the correct height and distance from players who will each have a different height and reach (Reimer \& Schwartz, 2016). As our sense of proprioception is disrupted in VR, it can be difficult to guess distances and the length of one's virtual arms (West, 2015). One solution is to support inaccurate interactions (Kroll, 2016), predicting the player's intentions throughout an interaction sequence (Whiting \& Donaldson, 2016).

\section{Controllers/Input}

Without any standard input system, players will need clear instructions that explain interactions in VR (Ludwig, 2013). As described in previous sections, any increase in realness will come with an increase in expectations: e.g., being able to pick up a cup with virtual hands often suggests to the player the ability to drink from it, pour liquid out, or break the cup (Schell, 2016). VR can leverage the way that users interact with the world on a daily basis, but designers must also be wary of any suggestion of 'intuitive' design, as the way that people interact with their surroundings is often idiosyncratic and dependent on a number of variables, such as their 
culture, age, and experience (Leap Motion, 2015). For now, 'natural' interactions in games are only semi-natural, which will have advantages and disadvantages for interaction design. In-game actions can be simplified so that players can repeat a task numerous times, but to some extent any action with a controller will also have to be learned (Bowman, 2016).

The input system will dictate the range of possible interactions in VR, both for the player and the designer. Head-tracking allows new gestures; for example, the player can select in-game options by looking, or nod or shake their head to respond to NPCs (Gage, 2016). Hand-tracked input with two controllers now allows players bi-manual interactions, i.e., actions that require two hands to perform (Malaika, 2015). But any physical input can be tiring or uncomfortable over time. Timed input like gaze-based interactions with head-tracking can be awkward, and pointing at or picking up objects with hand-tracked controllers can feel imprecise. The current solution is to provide generous active zones for hover-casting, and to allow for imprecise actions. Connecting two virtual objects might snap together (Podwal, Welker, Clement \& Tilton, 2016), or thrown objects might be more likely to hit their targets (Donaldson \& Whiting, 2016). As actions such as these are drawn from real life without exactly matching real life, players will need to be taught how and when they are able to interact.

\section{Learning How to Interact in VR}

Some actions and gestures appear to cross over nicely from other devices, such as resizing, scaling, or cloning objects (Podwal, Welker, Clement \& Tilton, 2016), but these and others will still require tutorializing, and it is useful to limit learned gestures. Leap Motion (2015) contends that users will only remember around five gestures, with the possibility of more when the basic gestures are combined. Because the controllers themselves are unfamiliar, some games include an in-game representation of the controller with text and visual cues introducing 
players to the button-mapping (West, 2015). When the controller is represented by virtual hands, the hands can communicate information about how to interact, again with visual cues or hand models mapped to a particular interaction (e.g., when grasping objects). The limited functionality of the virtual hand helps to constrain the player's expectations; similarly, the less realistic the hand, the less frustrating its limitations will be (Jahansson \& Huber, 2016). Overall, objects and interactions should have clear affordances, i.e., it is necessary to communicate what is possible or to anticipate potential interactions. For example, if an object has a handle, players may try to pull the handle. This is true for unplanned interactions as well. Schell (2016) suggests that when tasked with unscrewing a screw in the game, players might use the screwdriver, but might also use the knife. Both possibilities should be supported.

\section{UI/Menus}

Like other content, any conventional UI or menu will require redesign within a 360degree environment. Text in VR is a challenge, in part due to screen resolution, but also because many players simply will not read it (West, 2015). When necessary, use few words, increase the text size and position it at a comfortable distance, i.e., 2-3 meters from the player and within the middle 1/3 of their viewing area (Oculus, 2016a). The middle of the display is the least warped, which also implies that all traditional HUD elements in the corners of the screen are best when positioned elsewhere, ideally within 3D space (Callewaert, 2015). Curved menus can help to communicate depth, with content positioned to require minimal head movement, and even conventionally-styled menus can now exist within compelling animated worlds (Metcalfe, Alge, Faaborg, Dearman, \& Pullen, 2016). For the most part, UI and menus should strive to communicate information visually, using audio for additional support (Hamm \& Padget, 2016). 


\section{Diegetic UI}

Diegetic content refers to any content that exists within the space and time of the game world. When the UI exists within the world, some of the potential clutter of UIs can be removed while also helping to justify the placement and content of the interface (Doulin, 2016). There is a wide spectrum of diegetic content. A conventional interface positioned within the world could have diegetic shading and lighting to help integrate it in the environment (Metcalfe, Alge, Faaborg, Dearman \& Pullen, 2016). An object within the world could metaphorically represent an in-game action, for example a carabiner in The Climb (Crytek, 2016) represents a checkpoint (Kroll, 2016); in Job Simulator (2016) players insert cartridges for in-game choices, or eat a burrito to exit the game (Northway, 2016); in Cloudlands (Futuretown, 2016) players mini-putt towards a level choice (Northway, 2016); and in Fantastic Contraption (Northway Games \& Radial Games, 2016) an in-game helmet transports players to a menu world with model-sized versions of the levels. For more integrated diegetic content, Fantastic Contraption includes a companion cat character who acts as a physical embodiment of a selection menu (Voll, 2016a); or, in The Gallery (Cloudhead Games, 2016), a backpack positioned on the player's back acts as the player's inventory (Northway, 2016).

\section{Case Studies}

To better understand the practices outlined above, we created three interactive game-like prototypes for the Gear VR. Each game underwent iterative design and testing over the course of four months. We employed custom-designed qualitative and quantitative questionnaires based on standard usability questionnaires (e.g. Hart \& Staveland; Kennedy, Lane, Berbaum, \& Lilienthal, 1993) and modified to suit the focus of the design. Dionysian Dream VR tested side-scrolling and 
porting existing content to VR; Garden VR tested movement styles; and Donut Roller VR tested free-roam gameplay. Participants tested the various stages of Garden VR and Dionysian Dream $V R$ at RE/Lab (a total of 18 participants with two to five participants testing a particular iteration); with the results, a researcher at RE/Lab recommended changes and Phantom Compass iterated on the design. Time constraints prevented us from testing Donut Roller VR with participants. In this section we do not make any claim to the generalizability of our findings: with the variety of challenges in VR design, every new game will have to be modified to balance comfort and the desired player experience.

\section{VR Playtesting}

VR games require extensive and careful playtesting throughout their various stages of development to ensure player safety and comfort. VR is a medium that is unfamiliar to most players and most developers, with hardware and software that cannot mitigate all the negative side effects of VR. Developers will become increasingly poor judges of player comfort: first, because they will gradually acclimatize to the effects of VR (Lewis-Evans, 2014); and second, because when testing their own games their attention is inevitably drawn to details that the novice players may not focus on (Forsyth, 2014). Furthermore, during testing and during regular play, players will not necessarily know their own tolerance levels (Forsyth, 2014). Experiences designed for comfort should help players gain a literacy about VR, about their bodies, and about their level of comfort. Since self-reporting is not a completely reliable assessment, the use of additional questionnaires like the Simulator Sickness Questionnaire (SSQ) can be a good general addition (Oculus, 2016a).

Due to the several risks outlined in the Simulator Sickness and Player Comfort sections, playtesting must be conducted in safe and supervised environments (Johnson, 2016). Players 
should be aware of all the potential risks before consenting to test, with some review of the system before players begin the game. It is important to pay particular attention to warning signs, like sweating, excessive swallowing, pallor, belching (Forsyth, 2014). Some players may want to continue despite these symptoms, but sessions should be stopped at the first sign of discomfort. Players should also be made aware of the option to stop at any time. During playtesting, it is also important to watch how players move and perform any required gestures (Selan, 2015), both to learn how players use their bodies to play the game, but also to make sure players are safe.

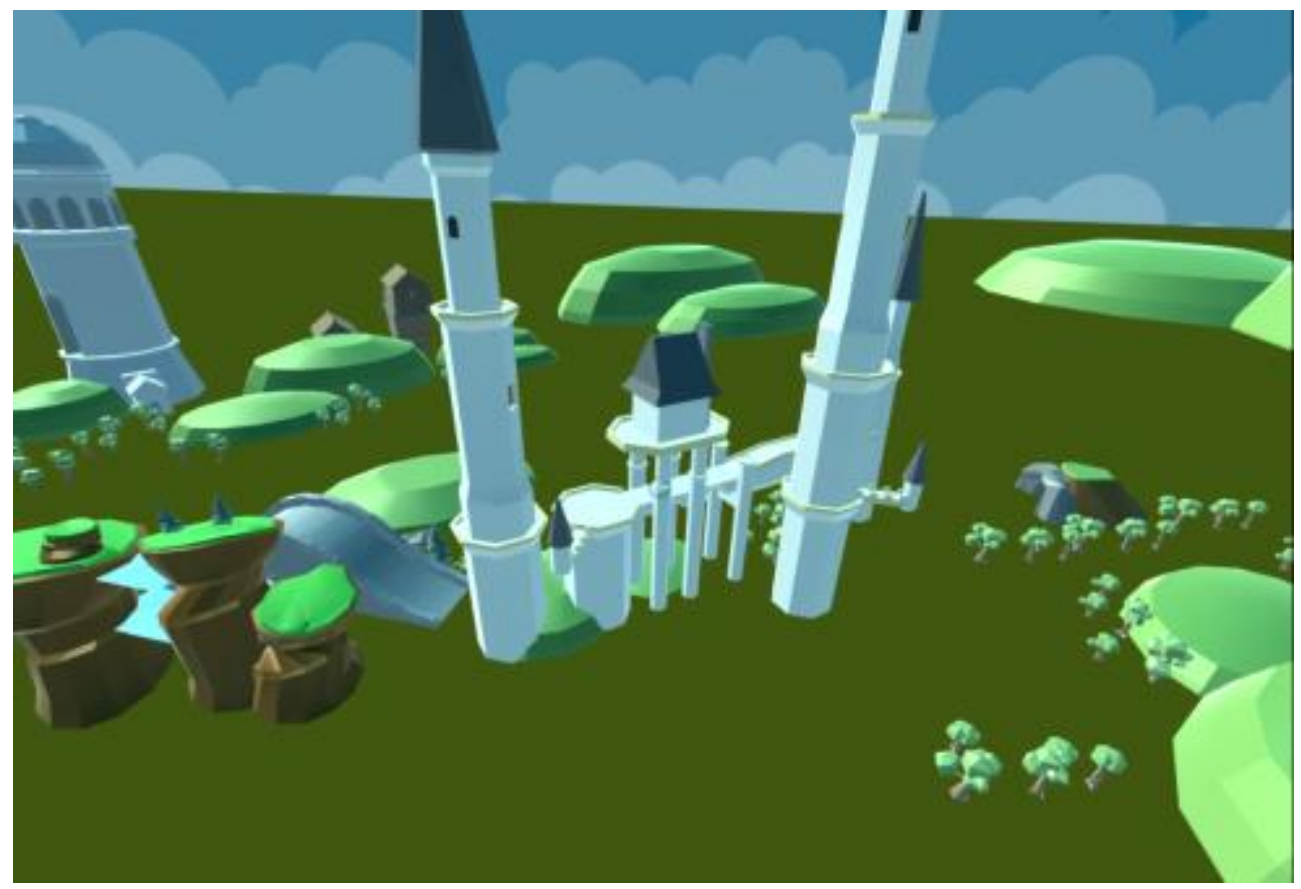

Figure 1. An early iteration of the Garden VR prototype. Players tested three separate movement styles when flying through the environment.

\section{Garden VR Prototype}

The goal of the Garden VR prototype (Figure 1) was to explore current design trends that strive to mitigate the discomfort of movement in VR. We experimented with teleportation, headsteering, and a grappling hook. Although teleportation is generally considered the most 
comfortable movement choice for VR, our participants reported that head-steering was the most comfortable, stating that it was easier to understand and that it offered more control. However, this is not a complete solution: further changes to the other forms of movement could have improved their results. With the grappling hook, players requested the ability to stop their movement at any time; with teleportation, players asked for a clearer indication of their destination point. Both options could also have benefitted from more visual cues before and during movement.

The testing helped to confirm many lessons learned from current best practices, for example, keeping the brightness low, or providing a stable skybox. Players requested configurable options, particularly with regard to movement speed. Current practices also

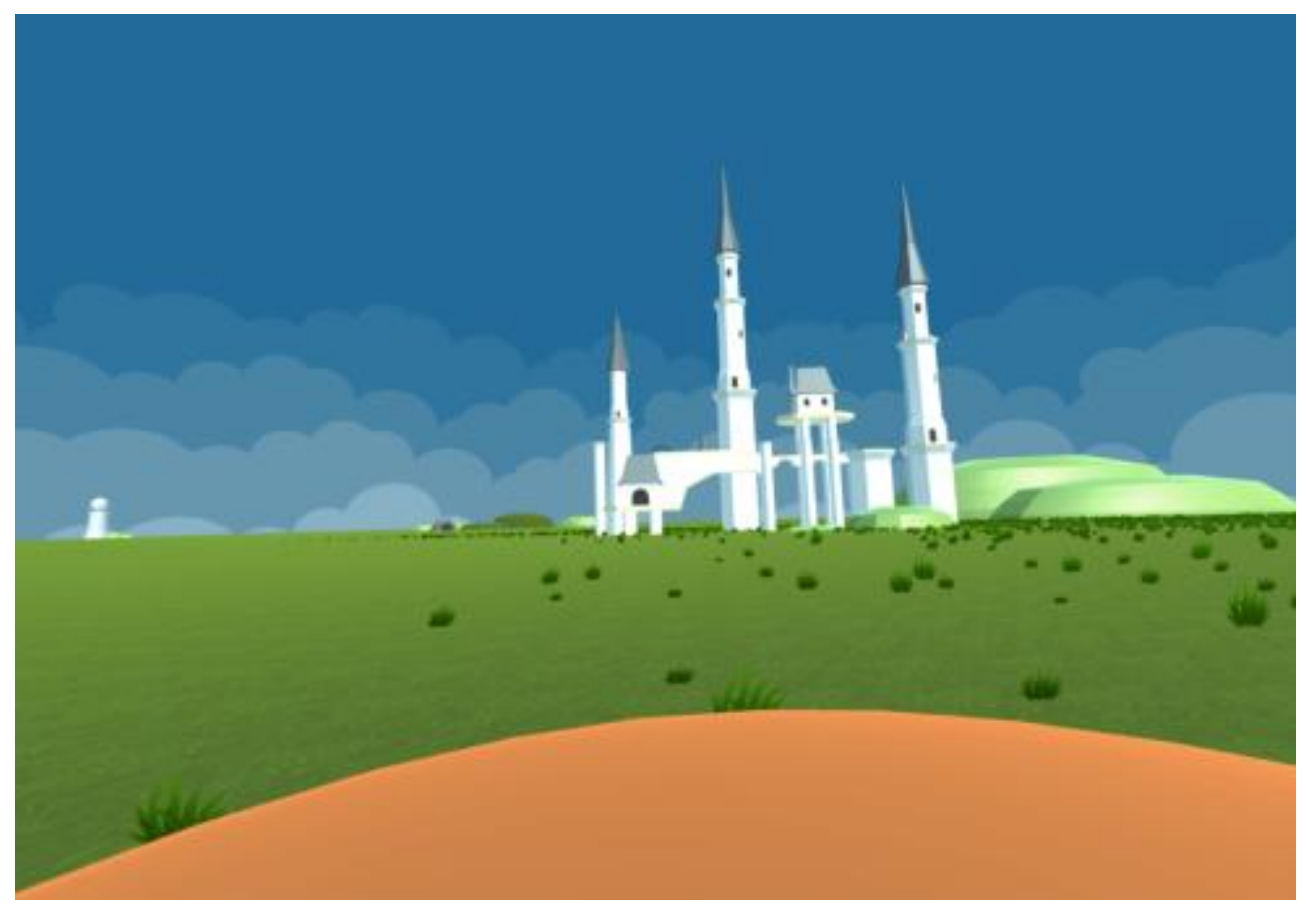

Figure 2. A later iteration with of the Garden VR prototype, using a flying saucer as stable reference point in the foreground. 
recommend providing a static reference point in the foreground of movement-based games, usually in the form of a cockpit or some other section of a vehicle. The responses to our addition of a flying saucer for players to sit on (Figure 2) seemed to reinforce this recommendation, but further testing would have been needed for the size and type of object. As an informal indication of progress, towards the end of the process players began requesting aesthetic changes, rather than those directly related to comfort.

\section{Dionysian Dream VR Prototype}

While the current generation of VR games suggests that cockpit-style games (e.g., in spaceships or cars) with exclusively forward movement are the most suited for VR, there are several genres and styles that have not yet been tested. The Dionysian Dream prototype (Figure 3) served two functions: first, experimenting with the conversion of an existing game into VR; and second, testing the side-scrolling format in VR. Our preliminary tests with Dionysian Dream

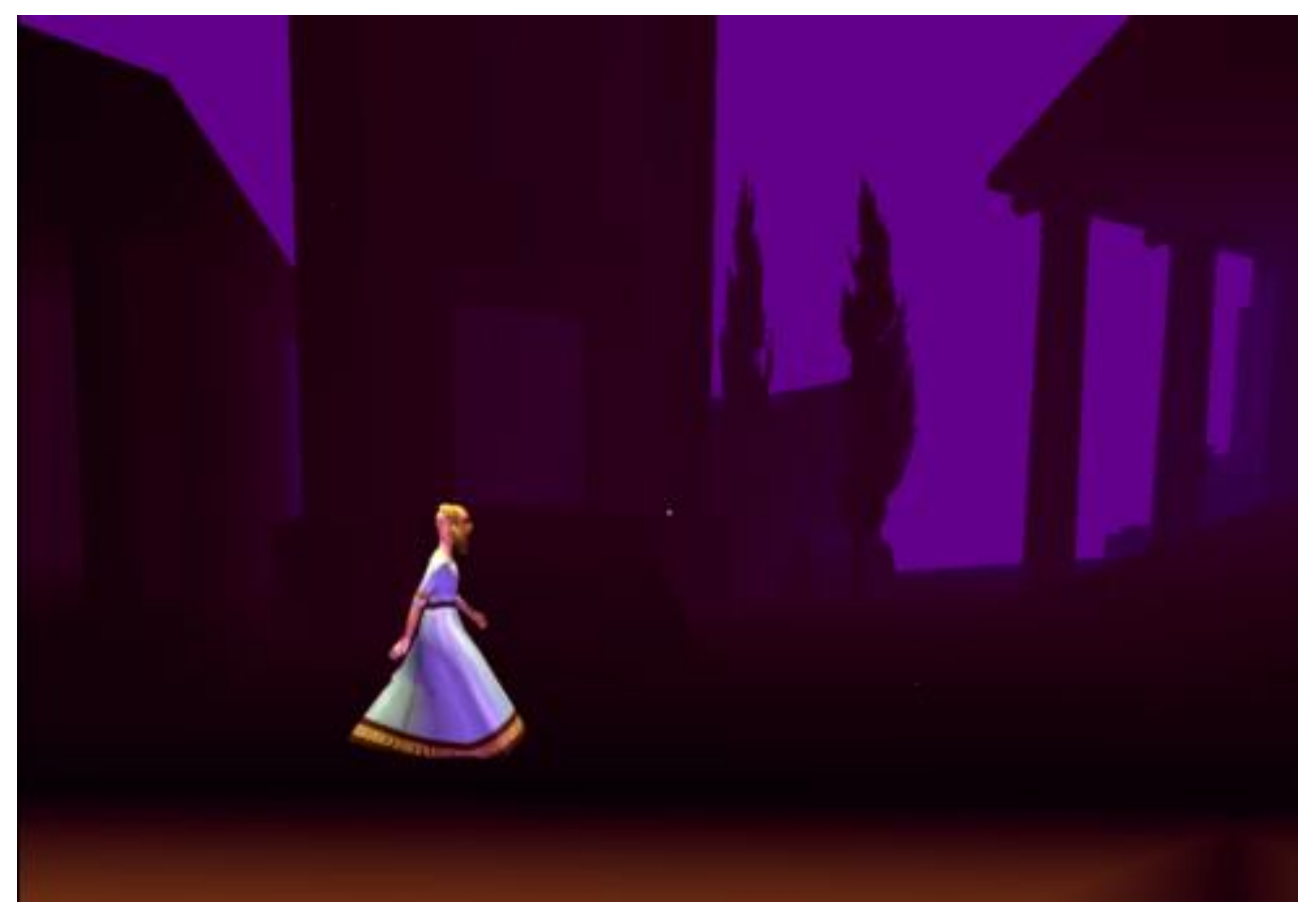

Figure 3. An iteration of the Dionysian Dream VR prototype. Players move the avatar right or left by looking ahead or behind the avatar. 
suggest that the side-scrolling genre is well suited to VR, with players widely reporting that the experience was comfortable. We suspect that this may be due to the relatively consistent movement speeds, the player's full control of the movement, and the camera position appearing fixed while the background scrolls to the left or right.

The text underwent many changes: by our final prototype the text had been enlarged, positioned to be stereoscopically closer and just below the centre of the screen (to prevent obstructing the view of the avatar), and set to auto advance with the voice-over (Figure 4).

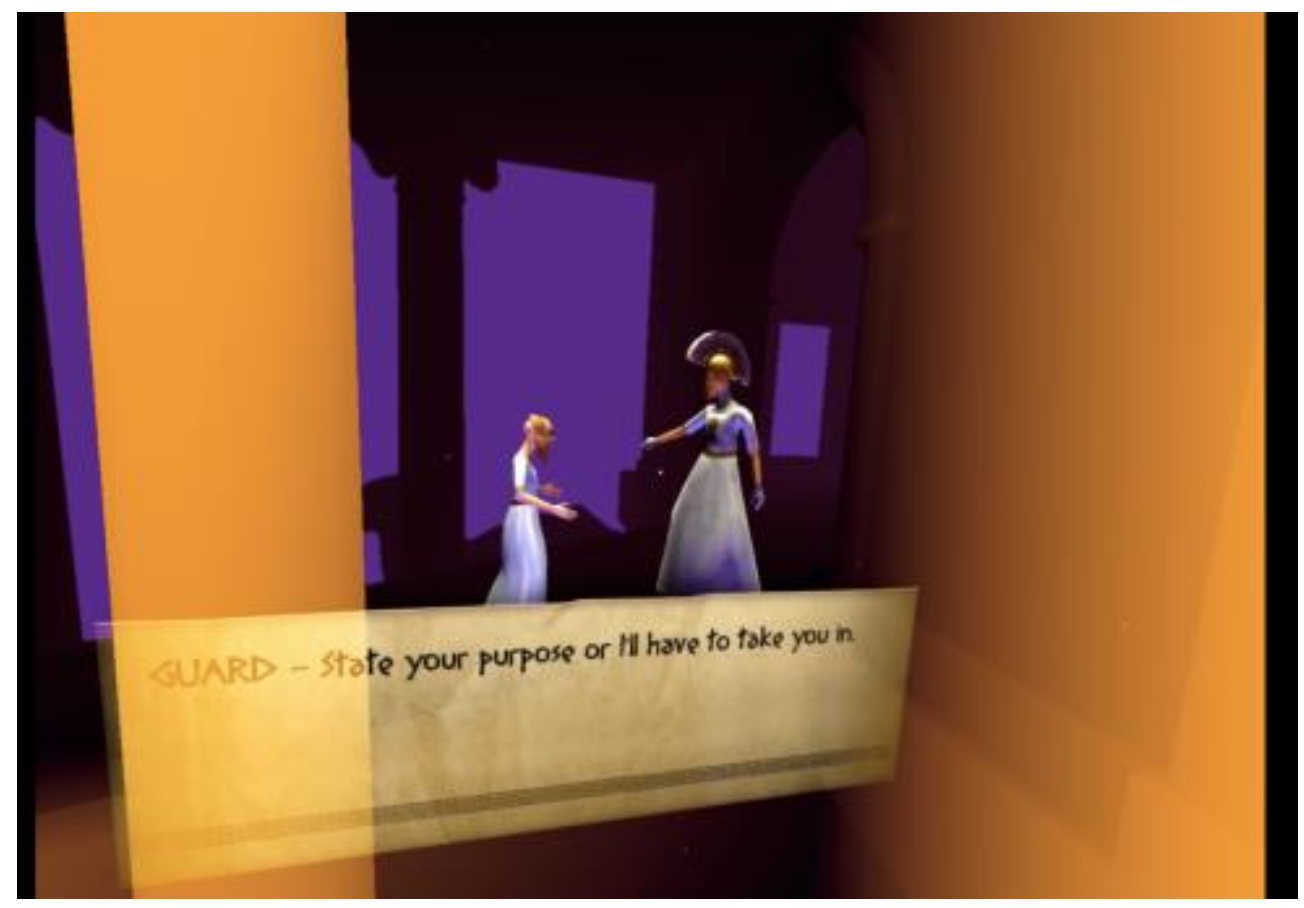

Figure 4. One of the final iterations of the text, now auto advancing and positioned just below the characters in order to keep the text and characters within the same view.

\section{Donut Roller VR Prototype}

Due to potentially uncomfortable camera movement, free-roaming games with a $3^{\text {rd }}$ person viewpoint are difficult in VR. In the Donut Roller prototype (Figure 5), the player rolls a ball through the streets of a miniature town. The game uses the $3^{\text {rd }}$ person viewpoint to 
experiment with solutions to the following questions: 1) How should the camera follow a ball that can travel to any point on the map, in any direction? 2) How does the player control the ball's movement, direction, speed? 3) How does the camera and ball react to in-game objects, collisions?

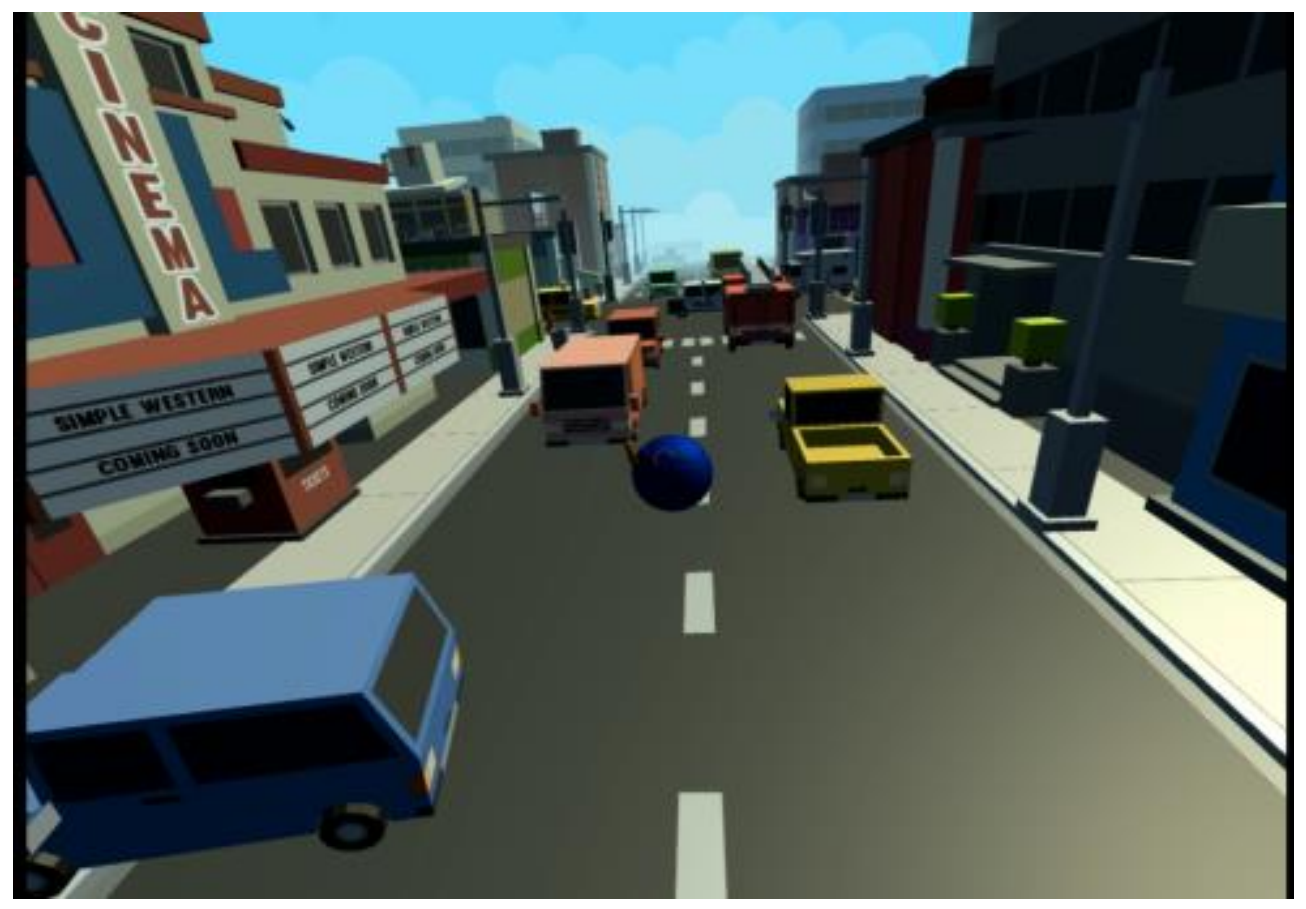

Figure 5. The Donut Roller VR prototype. All cars, trucks, and lampposts are destructible, resulting in many uncomfortable stops and starts in VR.

In our two iterations of the prototype, we referred to the Oculus Best Practices guide (2016) to identify several aspects that would require fixes in future iterations. These are in addition to standard changes like optimization for Gear VR, and in-game fixes. Many of the necessary changes could easily be implemented to improve the experience. For example, ensuring that the horizon line remain stable, maintaining player control of the camera, or decreasing the brightness. Other changes reflect the difficulty of free-roaming games in VR. For example, to mitigate the mismatch caused by the acceleration and the halted speed when colliding with objects, perhaps the speed could remain consistent, with collisions affecting in- 
game objects but not affecting the camera. Changes in direction could employ a dynamically reduced FOV, or perhaps fast snap turns. Overall, however, solutions to the above questions would require more extensive testing.

\section{Future Work}

Gamers and game designers are the first test subjects of VR (Davis, Darnell, Planck, Spiteri \& Bredow, 2016), but there are several potential VR markets, with predictions that less than half of VR will be games in the coming years (Carmack, 2015). With investments from Facebook and Google, there is also an emphasis on the social aspects of VR and its implied cinematic applications, with 360 YouTube and Facebook videos. For these applications to achieve widespread appeal, many of the factors noted throughout this document will have to be addressed. The changing hardware, expanding experiences and the unknown market potential of VR suggest that its best practices will have to be revisited over time (Montoya \& Sproll, 2016).

There is currently no consistency to VR experiences, and it is unknown how new audiences will respond to VR. For example, as of this writing Oculus does not recommend its headsets for children under thirteen, in part because young children are in a "critical period of visual development" (Oculus, 2016b), but Google has been using Cardboard for children and education with a reported one million students over the course of a year (Schrom, Holland, \& Jagnow, 2016). Meanwhile, the competition between hardware manufacturers affects the design parameters for each of the platforms as the shape, ergonomics, and input styles of each controller is different (Reimer \& Schwartz, 2016). For designers and developers seeking to reach wider audiences, designing games for multiple platforms may sacrifice what is unique to a single platform (McNeill, Kane, Hart, Geiger \& Link, 2015). Design that takes full advantage of room tracking with the Vive system will not be possible for the seated experiences of the Rift; or, 
design for tracked controllers and high-end headsets will lack the potentially wider user base of the mobile VR market (McNeill, 2016).

The rules of virtual worlds do not have to comply with our own: the geometry and physics of the world, as well as the appearance and capabilities of player avatars are all variables that can change from experience to experience. For developers, rapid prototyping and playtesting is necessary to keep abreast of trends while also pushing the boundaries of the medium. As players and developers expand the experiences of VR, its best practices will also expand. There is still no consensus on what VR as a medium is or can be. With its changing parameters, developers will have to learn and relearn the rules of VR, with each reassessment of the best practices beginning with player comfort. 


\section{References}

Abrash, M. (2015). Virtual Reality: the opportunities are now. [Video file]. Retrieved from https://www.youtube.com/watch?v=gJzgllp2zyc

AltspaceVR Inc. (2016). Introducing space bubble. [Blog post]. Retrieved from http://altvr.com/introducing-space-bubble/

Bowman, D. (2016). In 15 years of lessons from IEEE VR. [Video file]. Retrieved from http://www.gdcvault.com/play/1023647/15-Years-of-Lessons-From

Brillhart, J. (2016). VR \& cinema. [Video file]. Retrieved from https://www.youtube.com/watch?v=t3xDgONMdlM

Callewaert, C. (2015). VR game design advice, optimization, best practices. [Video file]. Retrieved from https://www.youtube.com/watch?v=ewWfQB9zdKA

Carmack, J. (2015). Oculus Connect 2 keynote. [Video file]. Retrieved from https://www.youtube.com/watch?v=Ti_3SqavXjk

Carmack, J. (2015). Public VR Critique \#5: Bazaar. [Blog post]. Retrieved from https://developer3.oculus.com/blog/public-vr-critique-5-bazaar/

Cloudhead Games. (2016). Call of the Starseed, The Gallery Episode 1 [Video game]. Retrieved from http://www.thegallerygame.com/blog/

Colgan, A. (2015). 5 experiments on the bleeding edge of VR locomotion. [Blog post]. Retrieved from http://blog.leapmotion.com/5-experiments-bleeding-edge-vr-locomotion/

Crytek. (2016). The Climb [Video game]. Retrieved from http://www.theclimbgame.com/

Davis, R., Darnell, E., Planck, M., Spiteri, N., \& Bredow, R. (2016). The future of VR. [Video file]. Retrieved from https://www.youtube.com/watch?v=Vjhcc-qKUJM

Doulin, A. (2016). Virtual reality development tips. [Video file]. Retrieved from http://www.gamasutra.com/blogs/AlistairDoulin/20160614/274884/Virtual_Reality_Deve lopment_Tips.php

Duringer, T. (2016). In VR microtalks: intersections of design \& technology. [Video file]. Retrieved from https://youtu.be/VtCuLd3wpEw?t=2355 
Ekanayake, C. (2016). Tiny worlds: table top scale in VR. [Video file]. Retrieved from https://youtu.be/VtCuLd3wpEw

Faliszek, C. (2015). The year ahead for HTC Vive Steam VR. [Video file]. Retrieved from https://www.youtube.com/watch?v=o8Ea6NqxnY4

Forsyth, T. (2014). Developing VR experiences with the Oculus Rift. [Video file].

Retrieved from https://www.youtube.com/watch?v=addUnJpjjv4

Foster, J. (2015). Sony on designing for believability for Morpheus VR. [Video file]. Retrieved from https://www.youtube.com/watch?v=whH3eVDz95o

Futuretown. (2016). Cloudlands [Video game]. Retrieved from http://futuretown.io/press/projects/cloudlands/

Gage, S. (2016). Building better stories in VR. [Video file]. Retrieved from https://www.youtube.com/watch?v=DD3WrkWH6jo

Gaudechon, J-C., \& Liu, K. (2015). Gunjack: the making of a Gear VR game. [Video file]. Retrieved from https://www.youtube.com/watch?v=Xs-0gisHoZA

Hamm, V. \& Padget, B. (2016). Pillars of presence: amplifying VR immersion. [Video file]. Retrieved from https://www.youtube.com/watch?v=TdJf3X5tBvU

Harris, P. (2015). Game design un-rules: examining design failures in Time Machine VR. [Video file]. Retrieved from https://youtu.be/14p9mqs2LiQ

Hart, S. G., \& Staveland, L. E. (1988). Development of nasa-tlx (task load index): Results of empirical and theoretical research. Human Mental Workload, 1, 139-183.

Heller, S. (2015). Beyond gaming: VR for the rest of the world. [Video file]. Retrieved from https://www.youtube.com/watch?v=LrcmCk-gupA

Hurd, D., \& Reiland, E. (2016). Lucky's Tale: the unexpected delight of third-person virtual reality, a technical postmortem. [Video file]. Retrieved from https://www.youtube.com/watch?v=J7L2SPZGbZg

Jahansson, J., \& Hubner, K. (2016). Enabling hands in virtual reality. [Video file]. Retrieved from https://www.youtube.com/watch?v=7i1DhIOhb5Y

Job Simulator. (2016). [Video game]. Retrieved from http://jobsimulatorgame.com/ 
Johnson, D. (2016). Best Practices for Introducing VR to New Users. Retrieved from http://uploadvr.com/best-practices-introducing-people-vr-first-time-new-users/

Joudrey, J. (2016). Knowing when to break the rules. [Video file]. Retrieved from https://www.youtube.com/watch?v=a97cbvriCpU

Kennedy R.S., Lane N.E., Berbaum K.S., Lilienthal M.G. (1993) Simulator sickness questionnaire: an enhanced method for quantifying simulator sickness. The international journal of aviation psychology, 3(3), 203-220.

Kroll, R. (2016). How Crytek builds 3-dimensional UI for VR. [Video file]. Retrieved from https://www.youtube.com/watch?v=Sq9NOukgxQc

Leap Motion. (2015). Leap Motion VR best practices guidelines. Retrieved from https://developer.leapmotion.com/assets/Leap\%20Motion\%20VR\%20Best\%20Practices $\%$ 20Guidelines.pdf

Lewis-Evans, B. (2015). Designing to minimize simulation sickness in VR games. [Video file]. Retrieved from https://www.youtube.com/watch?v=2UF-7BVf1zs

Link, H., \& Vasconcellos, N. (2016). Postmortem: bringing Adventure Time to virtual reality. [Video file]. Retrieved from https://www.youtube.com/watch?v=Udu0z0FKyqk

Luckey, P. (2014). Porting games to virtual reality. [Video file]. Retrieved from https://www.youtube.com/watch?v=-YCBadIVro8

Ludwig, J. (2013). What we learned porting Team Fortress 2 to virtual reality. [Video file]. Retrieved from https://www.youtube.com/watch?v=Gpr0FE2ATaY

Malaika, Y. (2015). Interaction design in VR: Valve's lessons. [Video file]. Retrieved from https://www.youtube.com/watch?v=_vQo0ApkAtI

Martz, N. (2016). VR for everyone on everyday phones. [Video file]. Retrieved from https://www.youtube.com/watch?v=K7teQZXugAI

McNeill, E. (2016). Two games, four platforms: a VR platform comparison. [Video file]. Retrieved from https://www.youtube.com/watch?v=hbybtv0isxE

McNeill, E., Kane, B., Hart, V., Geiger, M. \& Link, H. (2015). VR for indies. [Video file]. Retrieved from https://www.youtube.com/watch?v=kEL1kzul0ZE 
Metcalfe, L., Alge, M., Faaborg, A., Dearman, D., \& Pullen, B. (2016). Designing for Daydream. [Video file]. Retrieved from https://youtu.be/00vzW2-PvvE

Meyer, F. (2016). Adding natural motion to VR experiences. [Video file]. Retrieved from https://www.youtube.com/watch?v=IQHia15Ngmw

Montoya, D., \& Sproll, D. (2016). Don't just teleport - how to walk around something that is bigger than your tracked space. [Video file]. Retrieved from https://www.youtube.com/watch?v=At_Zac4Xezw

Northway, C. (2016). Fantastic Contraption and why VR menus suck. [Video file]. Retrieved from https://youtu.be/ASXST_iyhl4?t=19

Northway Games \& Radial Games (2016). Fantastic Contraption. [Video game]. Retrieved from http://northwaygames.com/presskit/sheet.php?p=contraption

Oculus. (2016a). Oculus Best Practices. Retrieved from http://static.oculus.com/documentation/pdfs/intro-vr/latest/bp.pdf

Oculus. (2016b). Health and Safety Warnings. Retrieved from https://www3.oculus.com/en-us/legal/health-and-safety-warnings/

O’Luanaigh, P. (2015). Controlling movement in virtual reality. [Video file]. Retrieved from https://www.youtube.com/watch?v=Zsg8L43k7QY

Podwal, M., Welker, S., Clement, M., \& Tilton, R. (2016). Daydream labs: lessons learned from VR prototyping. [Video file]. Retrieved from https://youtu.be/lGUmTQgbiAY

Reimer, D. \& Schwartz, A. (2014). Wild west of VR-discovering the rules of Oculus Rift development. [Video file]. Retrieved from https://www.youtube.com/watch?v=DqZZKi4UHuo

Reimer, D. \& Schwartz, A. (2016). Multiplatform VR - shipping a Vive, Oculus and Playstation VR title in Unity. [Video file]. Retrieved from https://www.youtube.com/watch?v=Xu7rmcudGNI

Riccitiello, J. (2016). Vision Summit 2016 Keynote. [Video file]. Retrieved from https://www.youtube.com/watch?v=ThpvQ9AwzrI 
Schell, J. (2016). I Expect You to Die: new puzzles, new hands. [Video file]. Retrieved from https://www.youtube.com/watch?v=X3Ad_OA7_SQ

Schrom, B., Holland, J., \& Jagnow, R. (2016). VR in the classroom: early lessons learned from Google Expeditions. [Video file]. Retrieved from

https://www.youtube.com/watch?v=UuceLtGjDWY

Shamlin, E. (2016). Adventures in cinematic VR: a year in review. [Video file]. Retrieved from https://www.youtube.com/watch?v=lxRxrRLEWow

Selan, J. (2015). Keeping an open mind in VR. [Video file]. Retrieved from https://www.youtube.com/watch?v=Y20twV0m_8

Thompson, L. \& Lloyd, G. (2016). Designing motion for vehicular VR games. [Video file]. Retrieved from https://www.youtube.com/watch?v=Gxj9Va6EYxI

Unity. (2016). Virtual Reality. [Tutorials] Retrieved from

https://unity3d.com/learn/tutorials/topics/virtual-reality

Unger, D. (2016). Stepping into VR locomotion. [Video file]. Retrieved from https://www.youtube.com/watch?v=mmv2uky4GhA

Unseld, S. (2015). 5 lessons learned while making Lost. [Blog post]. Retrieved from https://storystudio.oculus.com/en-us/blog/5-lessons-learned-while-making-lost/

Voll, K. (2016a). This is your brain on VR: a look at the psychology of doing VR right. [Video file]. Retrieved from https://www.youtube.com/watch?v=-owQfn-iYQw

Voll, K. (2016b). What people do in VR: designing believable experiences. [Video file]. Retrieved from https://www.youtube.com/watch?v=FMCiHiNBWgM

West, T. (2015). UX pointers for VR design. [Article]. Retrieved from https://medium.com/@timoni/ux-pointers-for-vr-design-dd52b718e19\#.votfiki8c

Whiting, N., \& Donaldson, N. (2016). Making Bullet Train and going off the rails in VR. [Video file]. Retrieved from https://www.youtube.com/watch?v=QIexGSksnPQ 